\title{
FATOS E TENDÊNCIAS DA BIOCATÁLISE
}

\author{
Caroline da C. S. Gonçalves e Anita J. Marsaioli* \\ Instituto de Química, Universidade Estadual de Campinas, Cidade Universitária Zeferino Vaz, 13081-970 Campinas - SP, Brasil
}

Recebido em 29/8/13; aceito em 13/9/13; publicado na web em 16/10/13

\begin{abstract}
BIOCATALYSIS: FACTS AND TRENDS. Brazil is renowned for its biodiversity; however, its economy is based on exotic plants, extraction and unsustainable use of natural resources. This issue was addressed in a recent QN review entitled "Chemistry without Borders." In order to explore the potential of Brazilian biodiversity fully, sustainable development is required in key technological areas, such as biotechnology. This research field is consistent with the green chemistry and white technology principles. Therefore, biotechnology is a sustainable alternative to conventional technologies and is expected to account for $20 \%$ of global chemicals by 2020. Brazil is the second largest grower of biotech crops and biodiesel, but its main activities rely on the fermentative process. In order to stimulate the national biotechnology development, the Brazilian Federal Government launched a national policy for biotechnology in 2007 and the National Committee of Biotechnology was created. Among the outstanding biotechnological processes, biocatalysis is one of the most important alternatives to conventional processing, and this field has changed dramatically with the advent of recombinant DNA technology in the 1970s, when large quantities of enzymes were accessible. The direct evolution methodology in the 1990s was a breakthrough and allowed tailoring of enzymes possessing high stability and stereoselectivity. However, about 60 years after the first industrial enzymatic biotransformation of steroids, the full potential of biocatalysis is far from being achieved. Future challenges in this field concern the multienzyme cascade reactions associated with optimized chemoenzymatic processes, and some recent industrial application of biocatalysts are also highlighted in this perspective.
\end{abstract}

Keywords: biotechnology; biocatalysis; multienzyme cascade reactions.

\section{INTRODUÇÃO}

O Brasil abriga uma das maiores biodiversidades do planeta, entretanto, grande parte das atividades econômicas nacionais é baseada em espécies exóticas, no extrativismo ou na utilização não sustentável destes recursos. Como apontado na revisão Química Sem Fronteiras $^{1}$ é preciso desenvolver uma visão estratégica que leve a um desenvolvimento sustentável, envolvendo as diversas áreas do conhecimento em prol da exploração consciente e efetiva do potencial da biodiversidade nacional. Dentre as fronteiras estratégicas necessárias para se alcançar este desenvolvimento, a biotecnologia merece destaque, pois é um campo dinâmico, multidisciplinar, intimamente relacionado a processos comprometidos com a química verde e que permite a união do economicamente viável com o ecologicamente correto. Estima-se que no ano de 2020 os processos biotecnológicos poderão estar envolvidos na manufatura de $20 \%$ dos produtos químicos, um mercado de bilhões de dólares. ${ }^{2}$

Dentre as diversas áreas biotecnológicas, a aplicação de processos enzimáticos é uma das alternativas mais promissoras para a substituição de metodologias convencionais. Atualmente, enzimas são empregadas industrialmente em diversos processos de produção como, por exemplo, produção de papel, tecidos, detergentes, alimentos, couro, cosméticos, biocombustíveis e produtos de química fina. As enzimas são altamente versáteis, podendo catalisar a transformação de substratos não naturais, de forma regio-, enantio-, e quimiosseletiva. As reações podem ser realizadas em temperatura ambiente e sobre pressão atmosférica, o que pode evitar a formação de subprodutos provenientes, por exemplo, de processos de isomerização e de rearranjos. Assim, a utilização de enzimas como biocatalisadores é bastante atrativa para a indústria farmacêutica, permitindo que intermediários quirais sejam obtidos

*e-mail: anita@iqm.unicamp.br por processos em sintonia com os preceitos da química verde e da biotecnologia branca, diminuindo-se a geração de resíduos e o consumo de energia. ${ }^{3}$

Neste artigo daremos, inicialmente, um panorama do setor biotecnológico do Brasil e do mundo e, em seguida, iremos abordar alguns tópicos recentes de pesquisas na área de biocatálise. Nossa intenção com este artigo é de apenas apontar para as futuras direções da biocatálise. Seria impossível cobrir toda a literatura disponível sobre o assunto, assim, antecipamos nossas desculpas aos pesquisadores que não forem mencionados.

\section{O CENÁRIO ATUAL DA BIOTECNOLOGIA NO BRASIL}

Dentre as 12 áreas tecnológicas consideradas estratégicas em 2012 as pesquisas em biotecnologia responderam por $4 \%$ do número total de patentes no mundo. ${ }^{4}$ No Brasil em 2010 o número de depósitos na área de biotecnologia no Escritório Europeu de Patentes foi 82\% superior ao ano $2000 .{ }^{5}$ Esta diferença é muito pequena, considerando dentre outros aspectos, o fato do país abrigar em torno de $15 \%$ do total de espécies existentes no planeta. A fim de estimular o desenvolvimento de biotecnologias inovadoras no Brasil, o governo federal criou no ano de 2007 o comitê Nacional de Biotecnologia e instituiu a Política de Desenvolvimento da Biotecnologia (decreto $N^{\circ}$ 6.041). O programa tem a meta ambiciosa de colocar o Brasil entre os cinco principais produtores de pesquisa, geração de serviços e produtos biotecnológicos até 2022.

Atualmente os Estados Unidos concentram o maior número de empresas biotecnológicas do mundo, seguido por países da Europa. O setor biotecnológico do Brasil é relativamente recente, sendo representado por apenas 237 empresas privadas, menos de $4 \%$ do número total de empresas do setor dos EUA em 2010. Entretanto, o Brasil ocupa uma posição de destaque nos setores de biocombustíveis e na produção de culturas biotecnológicas e é o maior exportador de grãos de soja transgênica para a China. ${ }^{6}$ 
As indústrias de biotecnologia no Brasil são em sua grande maioria (80\%) micros ou pequenas empresas, mais da metade estão ou estiveram em algum momento associadas a uma incubadora e muitas dependem do financiamento público (FINEP, CNPq e BNDES). O estado de São Paulo concentra a maioria das empresas do setor (40,5\%) seguido de Minas Gerais (24,5\%) e Rio de Janeiro $(13,1 \%)$. Estas empresas atuam principalmente áreas de saúde humana $(39,7 \%)$, saúde animal $(14,3 \%)$, reagentes $(13,1 \%)$, agricultura $(9,7 \%)$, meio ambiente $(9,7 \%)$ e bioenergia $(5,1 \%)$. Outros setores, como companhias farmacêuticas e de bioinformática, respondem por $8,4 \%$. Deve-se salientar que esta divisão de áreas foi feita desconsiderando-se biocombustíveis de origem vegetal, o que aumentaria consideravelmente a participação do setor de bioenergia. ${ }^{7}$

A análise dos dados estatísticos apresentados nos mostra que, apesar do setor biotecnológico em áreas como bioenergia e agricultura estarem bastante avançados no Brasil, nos demais setores muito ainda precisa ser feito. Em relação à aplicação de processos enzimáticos no setor de química fina, por exemplo, estamos ainda bastante atrasados, nossas atividades são ainda muito baseadas em processos fermentativos.

\section{AVANÇOS NA APLICAÇÃO INDUSTRIAL DE PROCESSOS BIOCATALÍTICOS}

Nos últimos anos observa-se um emprego industrial crescente de processos biocatalíticos, principalmente em áreas como química fina e farmacêutica. Entretanto, após mais de 60 anos desde a primeira biotransformação industrial de enzimática de esteroides, estamos muito longe de explorar todo o potencial da biocatálise. As dificuldades na obtenção de quantidades suficientes das enzimas para aplicações práticas representaram um dos maiores entraves para consolidação inicial dos processos biocatalíticos. A grande reviravolta neste cenário ocorreu na década de 90, com o advento das metodologias de engenharia molecular, que permitiu a produção de proteínas de interesse em grande escala. ${ }^{8}$ Estas novas metodologias ampliaram as aplicações industriais da biocatálise na produção de insumos de alto valor agregado. ${ }^{9}$ Atualmente, tem-se acesso a um grande número de enzimas expressas heterologamente e as propriedades biocatalíticas destas podem ser modificadas pelo emprego de técnicas de evolução dirigida. As enzimas modificadas podem ser mais específicas, enantiosseletivas e estáveis ou suas atividades catalíticas alteradas de forma a aceitar diferentes substratos. ${ }^{10}$

Como exemplo de aplicação industrial da biocatálise, apresentamos o trabalho desenvolvido por Liang e colaboradores, que empregaram uma ceto reductase engenheirada para conversão enantiosseletiva da cetona $\mathbf{1}$ ao álcool $\mathbf{2}$, intermediário chave na síntese do fármaco antiasmático Singulair ${ }^{\circledR}(\mathbf{3}$, Figura 1$) .{ }^{11} \mathrm{O}$ processo biocatalítico desenvolvido pelo grupo mostrou-se robusto e eficiente, possibilitando a obtenção do álcool 2 em excelente rendimento (>95\%), elevado excesso enatiomérico (ee>99,9\%) e boa pureza química $(>98,5 \%)$, em uma escala de $200 \mathrm{~kg}$.

Em um segundo exemplo, abordaremos a integração da biocatálise e a catálise química tradicional na obtenção de compostos enantiomericamente puros de forma direta. ${ }^{12}$ Johannes G. de Vries e colaboradores desenvolveram uma rota simples, curta e eficiente para a preparação do ácido $(S)$-indolina-2-carboxílico (4) a partir da combinação de biocatálise e catálise homogênea (Figura 2). ${ }^{13} \mathrm{O}$ processo desenvolvido está sendo aplicado pela DSM Pharma Chemicals para a produção de 4 em escala de toneladas. Entretanto, vale ressaltar que no processo desenvolvido por de Vries as reações químicas e de biocatálise são realizadas em recipientes diferentes. A verdadeira integração (realização das duas etapas num único reator) não é possível em todos os casos, devido principalmente a estabilidade das proteínas. Acreditamos que um futuro próximo pesquisas integrando a catálise química com a biocatálise serão cada vez mais comuns e promissoras.<smiles>CC(=O)c1ccccc1CCC(=O)c1cccc(/C=C/c2ccc3ccc(Cl)cc3n2)c1</smiles>

1

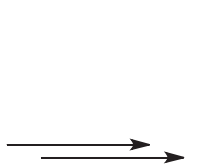<smiles>O=CCCCCCCCCl</smiles>

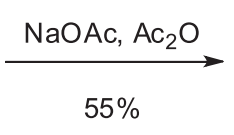

$55 \%$

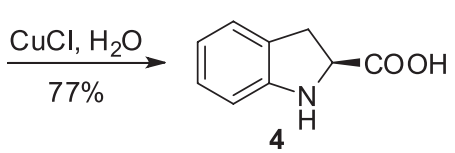<smiles>CC(=O)c1ccccc1CC[C@H](O)c1cccc(/C=C/c2ccc3ccc(Cl)cc3n2)c1</smiles>

2

$95 \%$ de rendimento ee $>99.9 \%$

Figura 1. Processo de biorredução enantiosseletiva da cetona 1 empregando uma ceto reductase engenheirada

5<smiles>O=Cc1ccccc1Cl</smiles><smiles>O=C(O)C=Cc1ccccc1Cl</smiles>

6<smiles>N[C@@H](Cc1ccccc1Cl)C(=O)O</smiles>

7

aração do ácido (S)-indolina-2-carboxílico (4) 


\section{NOVAS DIREÇÕES DA BIOCATÁLISE}

Os processos enzimáticos que eram limitados a uma única etapa de transformação estão sendo organizados de forma a gerar metabolismos artificiais (Systems biocatalysis - SysBiocat) ${ }^{14}$ nos quais biotransformações sequenciais formam produtos enantiomericamente puros. A utilização de biotransformações em cascata encontra-se em sintonia com os conceitos da química verde, pois evita o isolamento dos intermediários, minimiza a geração de resíduos, o consumo de energia e reduz o custo de produção. ${ }^{15}$

Dentro deste contexto, Kroutil e colaboradores realizaram a síntese diastereosseletiva de derivados de cicloexilaminas (8) a partir de dicetonas bicíclicas proquirais $(\mathbf{9})$, aplicando um processo multienzimático ( $\beta$-dicetona hidrolase, lipase e $\omega$-transaminase) em três etapas (Figura 3). ${ }^{16}$ A primeira etapa envolveu a hidrólise estereosseletiva de um uma ligação $\mathrm{C}$ - $\mathrm{C}$ catalisada por uma $\beta$-dicetona hidrolase (reação enzimática de retro-Claisen de $\beta$-dicetonas proquirais), sendo o $\beta$-cetoácido 10, obtido em elevado grau de pureza óptica. A segunda etapa envolveu a esterificação de $\mathbf{1 0}$ catalisada pela lipase B de Candida antarctica, entretanto, o composto 10c não foi aceito como substrato pela enzima e foi esterificado quimicamente. Finalmente, na terceira etapa, o segundo centro foi introduzido via uma reação de aminação catalisada por uma $\omega$-transaminase. Pela escolha da $\omega$-transaminase adequada foi possível produzir seletivamente os isômeros cis-8 ou trans-8 em elevado grau de pureza óptica $(e e>99 \%, d e=97$ a $99 \%)$.

Em uma terceira abordagem, Rother e colaboradores desenvolveram um processo multienzimático no qual o co-produto da segunda etapa é utilizado como substrato na primeira ("recycling cascade"). Este processo permitiu a obtenção da $(1 R, 2 R)$-norpseudoefedrina (11) e da $(1 R, 2 S)$-norefedrina (12) em bons rendimentos e elevada pureza óptica (Figura 4). ${ }^{17}$

\section{CONCLUSÃO}

A necessidade crescente de novos biocatalisadores e o advento de técnicas como engenharia molecular e metagenômica, demandam o desenvolvimento de metodologias rápidas para revelar as atividades enzimáticas (ensaios de triagem enzimática). Nas últimas décadas foram desenvolvidos vários ensaios de triagem enzimática baseados, por exemplo, na utilização de instrumentos analíticos (ex: HPLC, CG, MS e RMN), variações de $\mathrm{pH}$ do meio, na utilização de radioisótopos ou de substratos enzimáticos sintéticos (sondas enzimáticas). ${ }^{18}$ Nosso grupo de pesquisas vem trabalhando com a triagem e monitoramento das atividades enzimáticas de microrganismos e enzimas isoladas por mais de dez anos. ${ }^{19}$

Esperamos ter mostrados que a biotecnologia é uma ciência multidisciplinar, associada aos preceitos da química verde e que pode auxiliar na união do desenvolvimento econômico com o tão almejado desenvolvimento sustentável. Contudo, para transformamos o Brasil na quinta potência mundial do setor biotecnológico muito ainda necessita ser feito. Por exemplo, precisamos de leis mais claras, simples e melhores para o setor, as iniciativas de colaboração entre indústria e a academia devem ser fortalecidas e devemos aumentar os incentivos a programas de capacitação de mão de obra especializada e investir no empreendedorismo.<smiles>[R]C1C2C(=O)CC(C2=O)C1([R])[R]</smiles>

9a, $R^{1}, R^{2}=H$

$9 b, R^{1}=M e, R^{2}=H$

$9 c, R^{1}=H, R^{2}=M e$

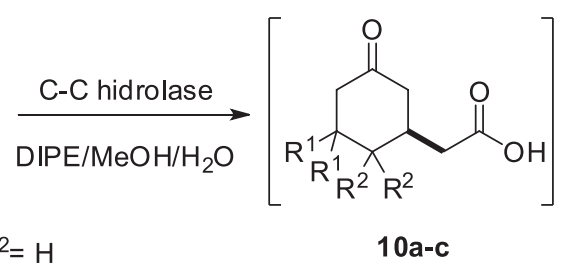

10a-c

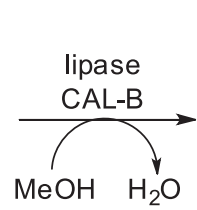

$\mathrm{MeOH} \quad \mathrm{H}_{2} \mathrm{O}$

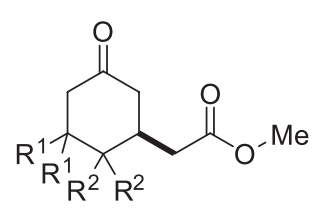

11a-c

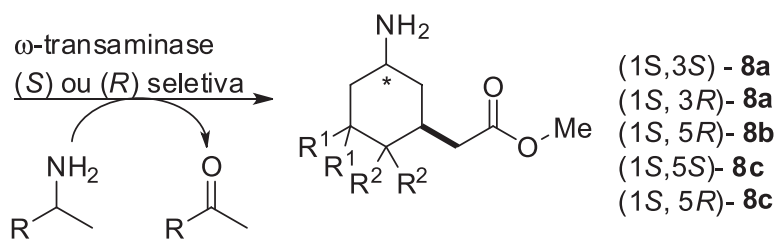

Figura 3. Processo multienzimático para obtenção de derivados de cicloexilaminas (8) a partir de dicetonas bicíclicas proquirais (9)

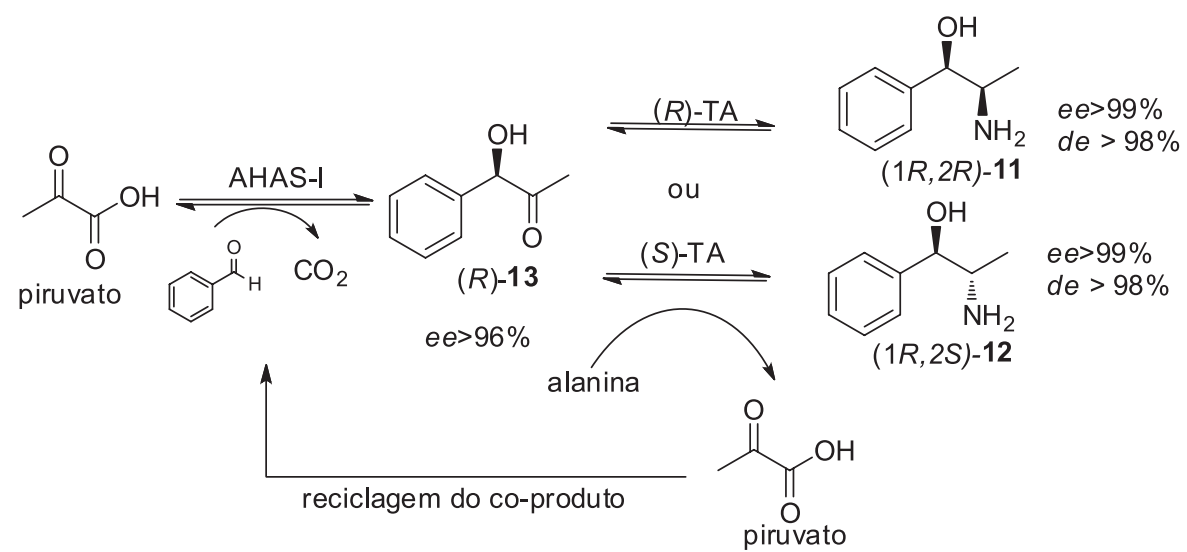

Figura 4. Processo multienzimático utilizado na síntese da norpseudoefedrina ((1R,2R)-11) e da norefedrina ((1R,2S)-12) 
Em relação à biocatálise, ao alcançarmos os sistemas biocatalíticos ou sistemas multienzimáticos é possível visualizar que a próxima barreira a ser vencida é a otimização de processos de interesse a partir da fusão da biocatálise com a química sintética e com a engenharia química.

\section{AGRADECIMENTOS}

Os autores agradecem as agências de fomento Petrobrás-ANP, CNPq e FAPESP, ao Instituto de Química - UNICAMP pelo suporte e aos editores da Quimica Nova pelo convite.

\section{REFERÊNCIAS}

1. Pinto, A. C.; Zucco, C.; Galembeck, F.; de Andrade, J. B.; Vieira, P. C.; Quim. Nova 2012, 35, 2092.

2. Meyer, H. -P.; Org. Process Res. Dev. 2011, 15, 180.

3. Jegannathan, K. R.; Nielsen, P. H.; J. Cleaner Prod. 2013, 42, 228.

4. http://thomsonreuters.com/press-releases/032013/759960, acessada em Agosto 2013

5. http://www.mct.gov.br/, acessada em Agosto 2013.

6. a)http://www.isaaa.org/resources/publications/biotech_country_facts_ and_trends/download/Facts\%20and\%20Trends\%20-\%20Brazil.pdf, acessada em Agosto 2013. b) http://www.biodieselbr.com/noticias/bio/ brasil-segundo-maior-produtor-biodiesel-030811.htm

7. http://www.cebrap.org.br/v1/upload/pdf/Brazil_Biotec_Map_2011.pdf, acessada em Agosto 2013.

8. Reetz, M. T.; J. Am. Chem. Soc. 2013, 135, 12480.

9. Turner, N. J.; O’Reilly, E.; Nat. Chem. Biol. 2013, 9, 285.

10. Woodley, J. M.; Curr. Opin. Chem. Biol. 2013, 17, 310; Reetz, M. T. Tetrahedron 2012, 68, 7530; Bornscheuer, U. T.; Huisman, G. W.; Kazlauskas, R. J.; Lutz, S.; Moore, J. C.; Robins, K.; Nature 2012, 485, 185; Hult, K.; Berglund, P.; Curr. Opin. Biotechnol. 2003, 14, 395; Jaeger, K. -E.; Reetz, M. T.; Curr. Opin. Chem. Biol. 2000, 4, 68.

11. Liang, J.; Lalonde, J.; Borup, B.; Mitchell,V.; Mundorff, E.; Trinh, N.; Kochrekar, D. A.; Cherat, R. N.; Pai, G. G.; Org. Process. Res. Dev. 2010, 14, 193.
12. Harles, H. C.; Dalby, P. A.; Woodley, J. M.; J. Chem. Technol. Biotechnol. 2007, 82, 1063.

13. de Lange, B.;Hyett, D. J.; Maas, P. J. D.; Mink, D.; van Assema, F. B. J.; Sereinig, N.; de Vries, A. H. M.; de Vries, J. G.; ChemCatChem 2011, $3,289$.

14. de Graaff C.; Ruijter, E.; Orru, R. V. A.; Chem. Soc. Rev. 2012, 41, 3969; Ruijter, E.; Scheffelaar, R.; Orru, R. V. A.; Angew. Chem. Int. Ed. 2011, 50,6234

15. Oroz-Guinea, I.; Gárcia-Junceda, E.; Curr. Opin. Chem. Biol. 2013 , 17, 236; Sánchez-Moreno, I.; Hélaine, V.; Poupard, N.; Charmantray, F.; Légeret, B.; Hecquet, L.; García-Junceda, E.; Wohlgemuth, R.; Guérard-Hélaine, C.; Lemaire, M.; Adv. Synth. Catal. 2012, 354, 1725; Schrittwieser, J. H.; Curr. Opin. Chem. Biol. 2011, 15, 249; Ricca, E.; Brucher, B.; Schittwieser, J. H.; Adv. Synth. Catal. 2011, 353, 2239.

16. Siirola, E.; Mutti, F. G.; Grischek, B.; Hoefler, S. F.; Fabian, W. M. F.; Grogan, G.; Kroutil, W.; Adv. Synth. Catal. 2013, 355, 1703.

17. Sehl, T.; Hailes, H. C.; Ward, J. M.; Wardenga, R.; von Lieres, E.; Offermann, H.; Westphal, R.; Pohl, M.; Rother, D.; Angew. Chem. Int Ed. 2013, 52, 6772 .

18. Goddard, J.-P.; Reymond, J.-L.; Curr. Opin. Biotechnol. 2004, 15, 314; Haugland, R. P.; Handbook of Fluorescence probes and Research chemicals, $6^{\text {th }}$ ed, Molecular Probes: Eugene, Oregon, 1996.

19. de Vasconcellos, S. P.; Angolini, C. F. F.; García, I. N. S.; Dellagnezze, B. M.; da Silva, C. C.; Marsaioli, A. J.; Santos Neto, E. V.; de Oliveira, V. M.; Org. Geochem. 2010, 41, 675; da Cruz, G.; Angoline, C. F. F.; de Oliveira, L. G.; Lopes, P. F.; de Vasconcellos, S. P.; Crespim, E.; de Oliveira, V. M.; Santos neto, E. V.; Marsaioli, A. J.; Appl. Microbiol. Biotechnol. 2010, 87, 319; Mantovani, S. M.; de Oliveira, L. G.; Marsaioli, A. J.; J. Braz. Chem. Soc. 2010, 21, 1484; Mantovani, S. M.; de Oliveira, L. G.; Marsaioli, A. J.; J. Mol. Catal. B: Enzym. 2008, 5253, 173; Pinheiro, L.; Marsaioli, A. J.; J. Mol. Catal. B: Enzym. 2007, 44, 78; Sicard, R.; Chen, L. S.; Marsaioli, A. J.; Reymond, J.- L.; Adv. Synth. Catal. 2005, 347, 1041; Bicalho, B; Chen, L. S.; Grognux, J.; Reymond, J.-L.; Marsaioli, A. J.; J. Braz. Chem. Soc. 2004, 15, 911; Gonçalves, R. A. C.; Porto, A. L. M.; Pinheiro, L.; Cagnon, J. R.; Manfio, G. P.; Marsaioli, A. J.; Food Technol. Biotechnol. 2004, 4, 355. 